\title{
upporting Information
}

\section{Mechanical Failure Mechanism of Silicon-based Composite Anodes under Over-Discharging Conditions Based on Finite Element Analysis}

Shugui Song, ${ }^{\dagger}$ Mingyun Zhu, ${ }^{\dagger}$ Yuwei Xiong,,$^{\dagger}$ Yifeng Wen,,$^{\dagger}$ Meng Nie ${ }^{\dagger}$, Xiangyu Meng, ${ }^{\S}$ Anqi Zheng, ${ }^{\dagger}$ Yongqiang Yang, ${ }^{*,+}$ Yunqian Dai, ${ }^{*} \S$ Litao Sun ${ }^{\dagger}$, and Kuibo Yin ${ }^{*},+$

${ }^{\dagger}$ SEU-FEI Nano-Pico Center, Key Laboratory of MEMS of Ministry of Education, Southeast University, Nanjing, Jiangsu 210096, P. R. China

National Graphene Products Quality Supervision and Inspection Center (Jiangsu), Jiangsu Province Special Equipment Safety Supervision Inspection Institute, Wuxi, Jiangsu 214174, P. R. China

${ }^{\S}$ School of Chemistry and Chemical Engineering, Southeast University, Nanjing, Jiangsu 211189, P. R. China

*Corresponding Author Email: Kuibo Yin, yinkuibo@seu.edu.cn; Yongqiang Yang, yqyang@wxtjy.com; Yunqian Dai, daiy@seu.edu.cn 

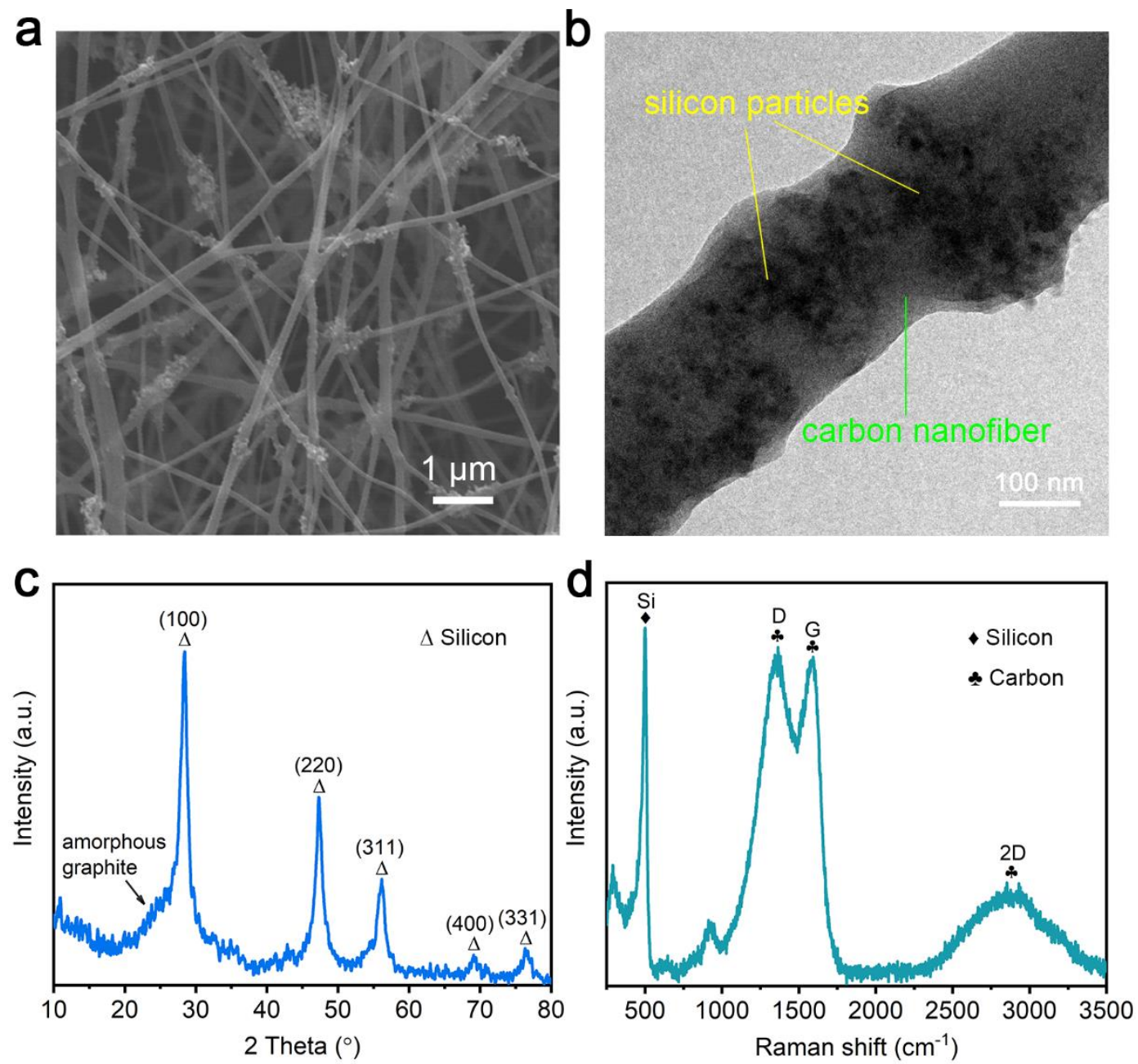

Figure S1. Microstructure characterization. (a) TEM image and (b) SEM image of Silicon/Carbon nanofibers (Si/CFs) composite. (c) XRD pattern and (d) Raman spectra of Si/CFs. 

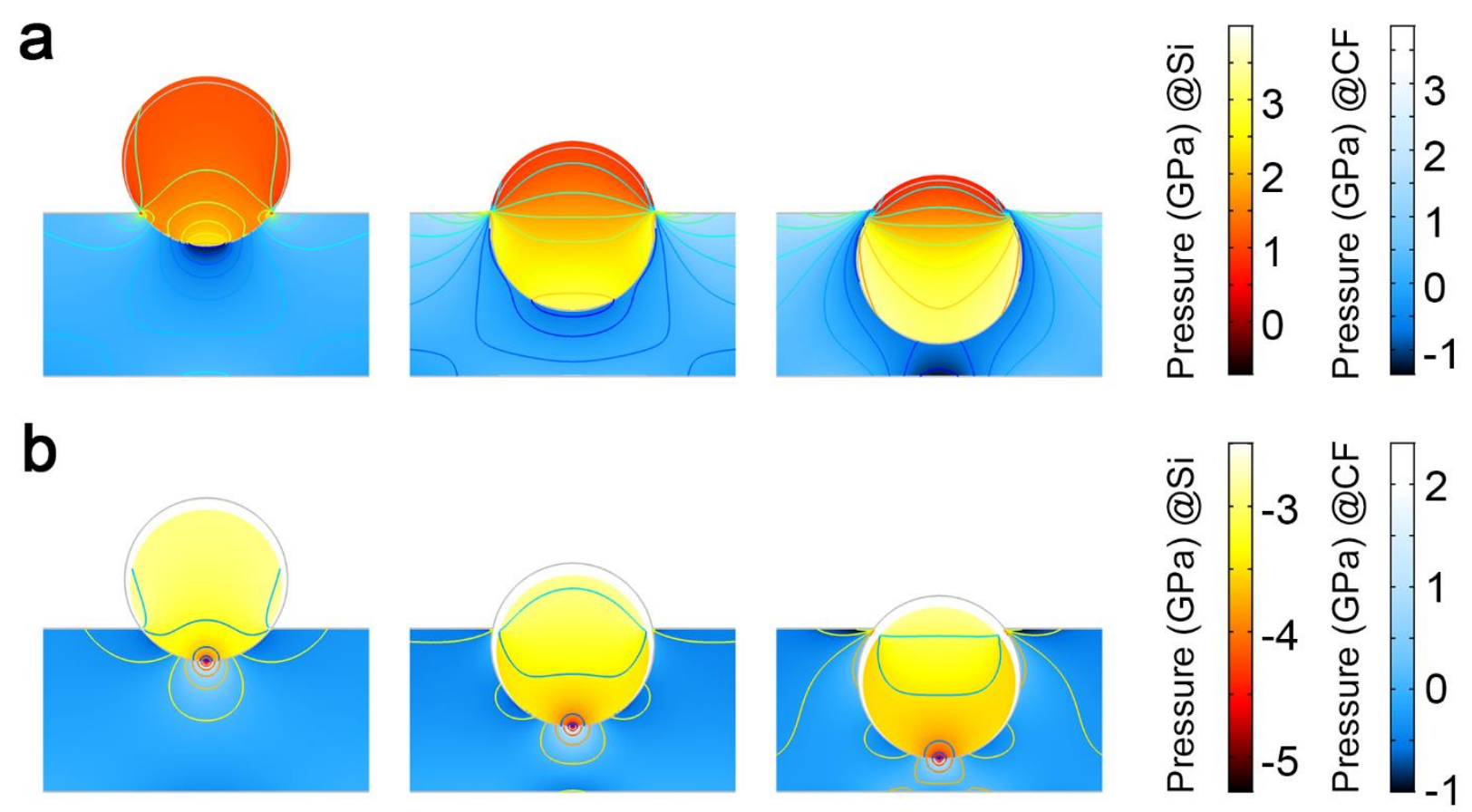

Figure S2. The distributions of pressure under (a) charging ( $\Delta \mathrm{T}=300 \mathrm{~K}$, similarly hereinafter) and (b) over-discharging $(\Delta \mathrm{T}=-600 \mathrm{~K}$, similarly hereinafter) conditions. 


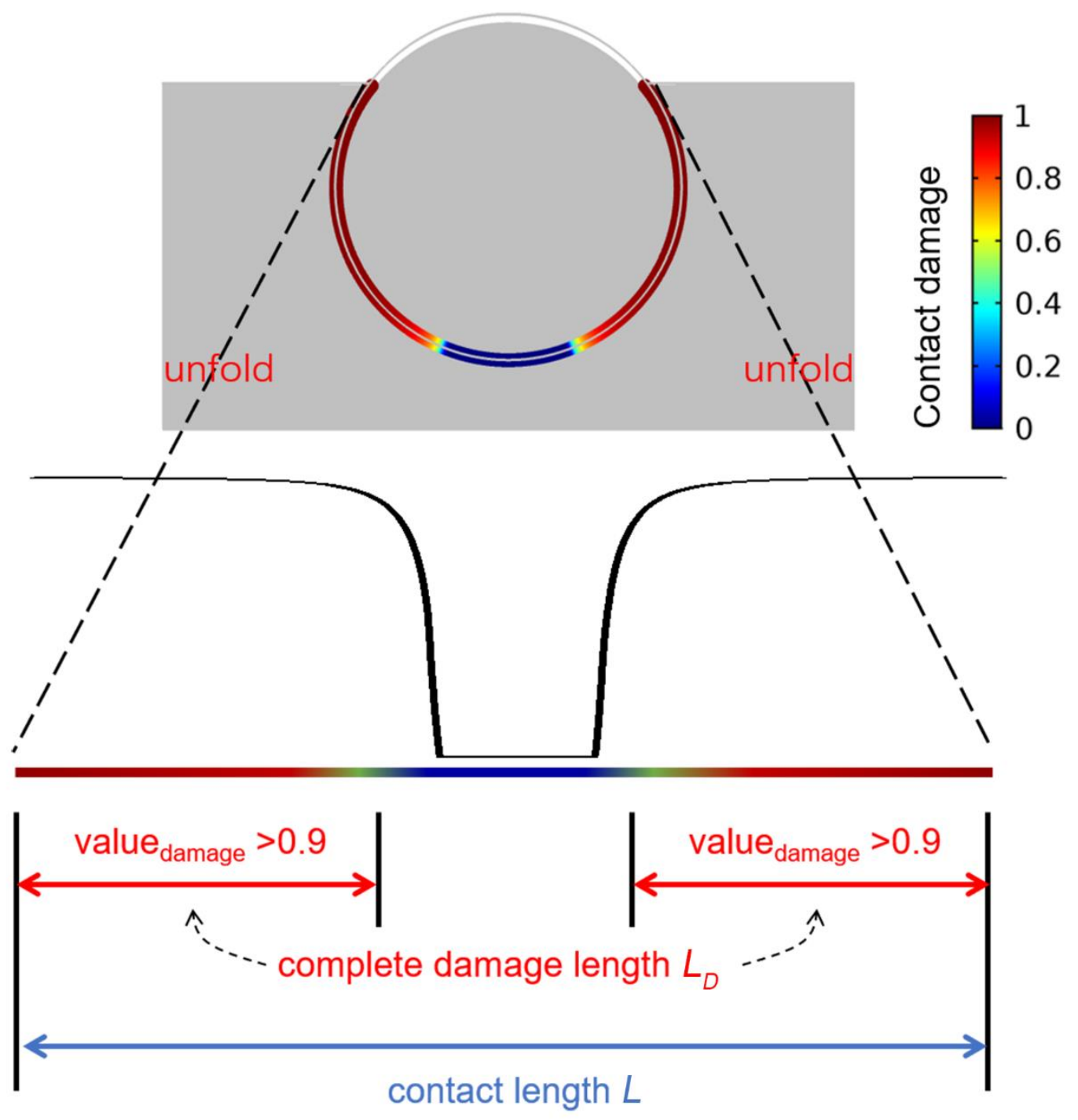

Figure S3. Schematic diagram of contact damage, contact length $(L)$, and complete damage length $\left(L_{D}\right)$. 


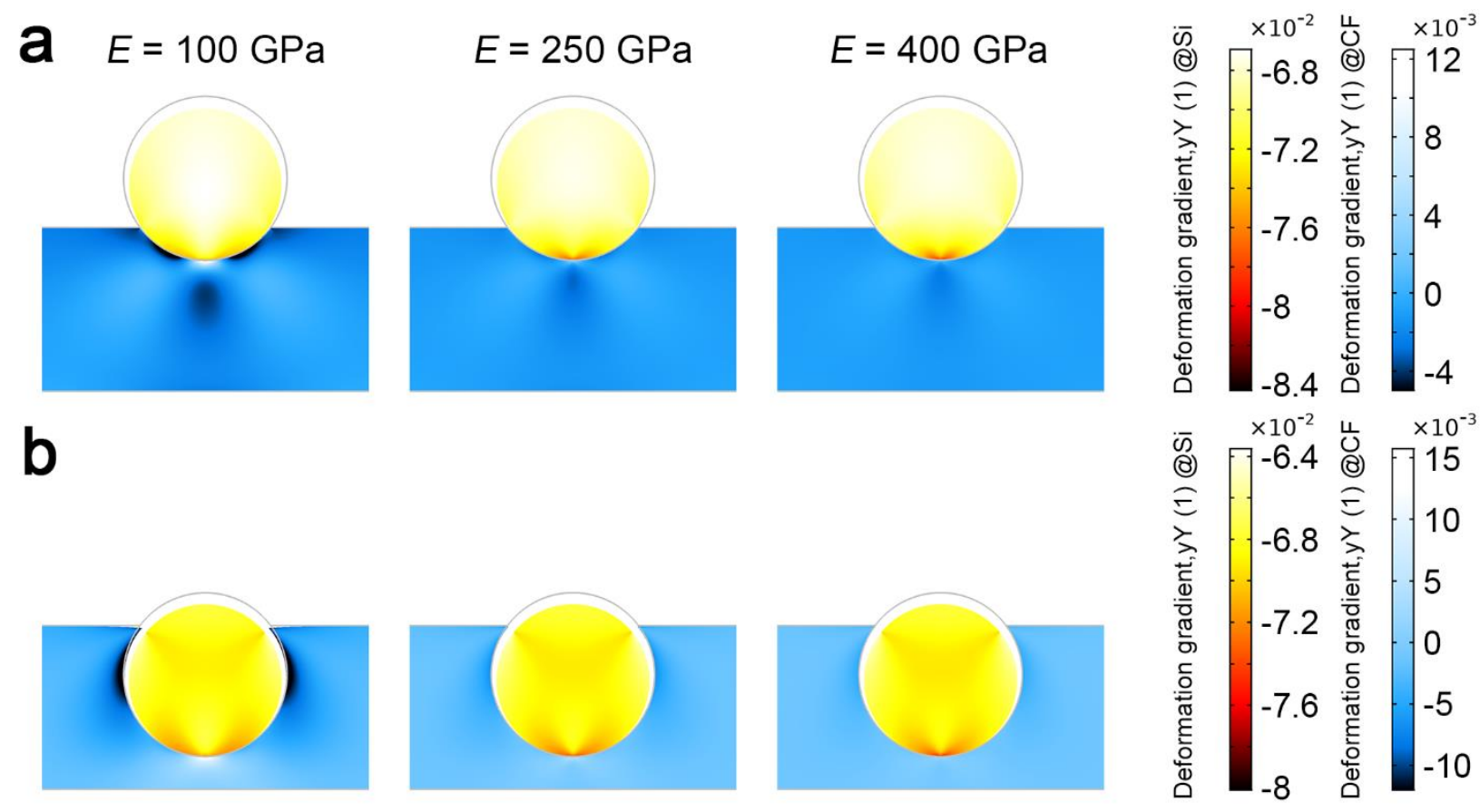

Figure S4. The distribution of deformation gradient (yY component) in (a) SC-L and (b) SC-H in over-discharging conditions under Young's modulus $(E)$ ranging from $100 \mathrm{GPa}$ to $400 \mathrm{GPa}$. 


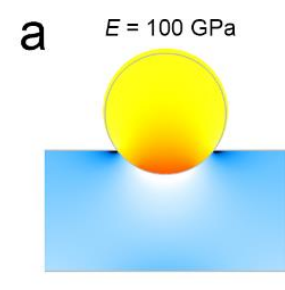

b

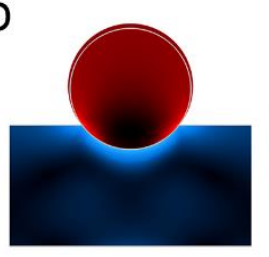

C

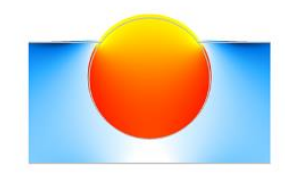

d

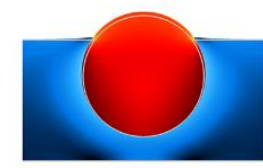

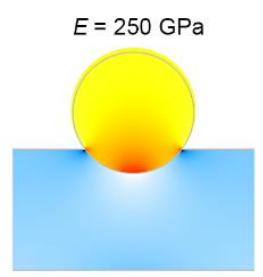
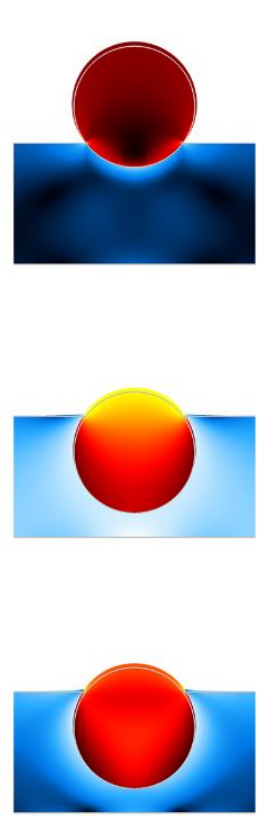
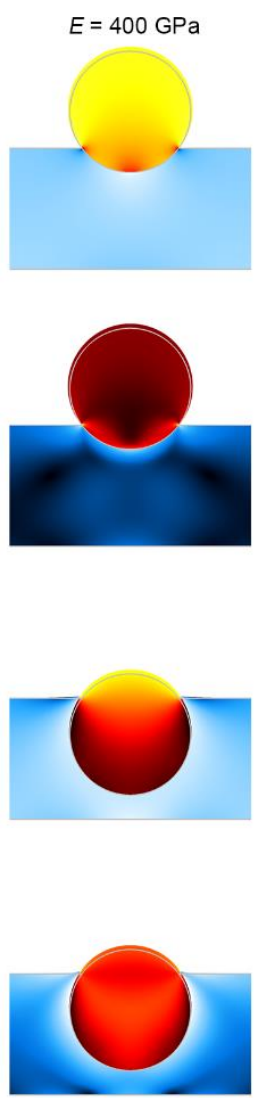
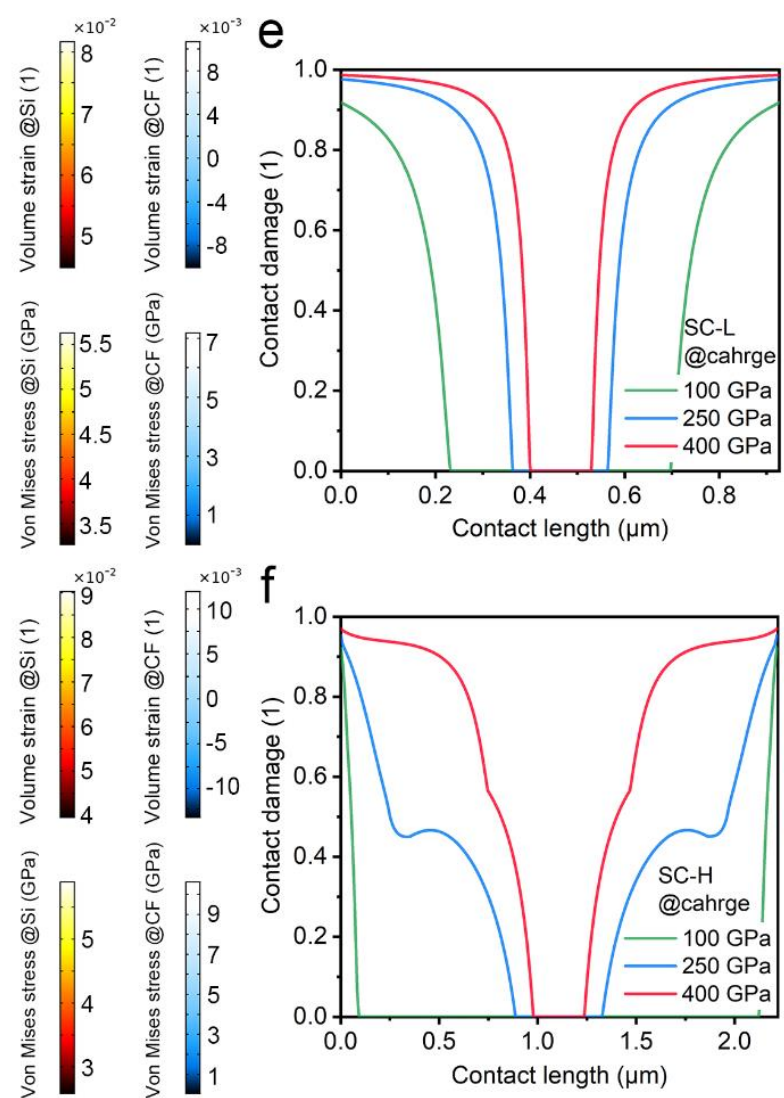

Figure S5. The distribution of volume strain in (a) SC-L and (c) SC-H, and the distribution of von Mises stress in (b) SC-L and (d) SC-H in charging conditions under Young's modulus (E) ranging from $100 \mathrm{GPa}$ to $400 \mathrm{GPa}$. Contact damage of (e) SC-L and (f) SC-H under charging conditions. 


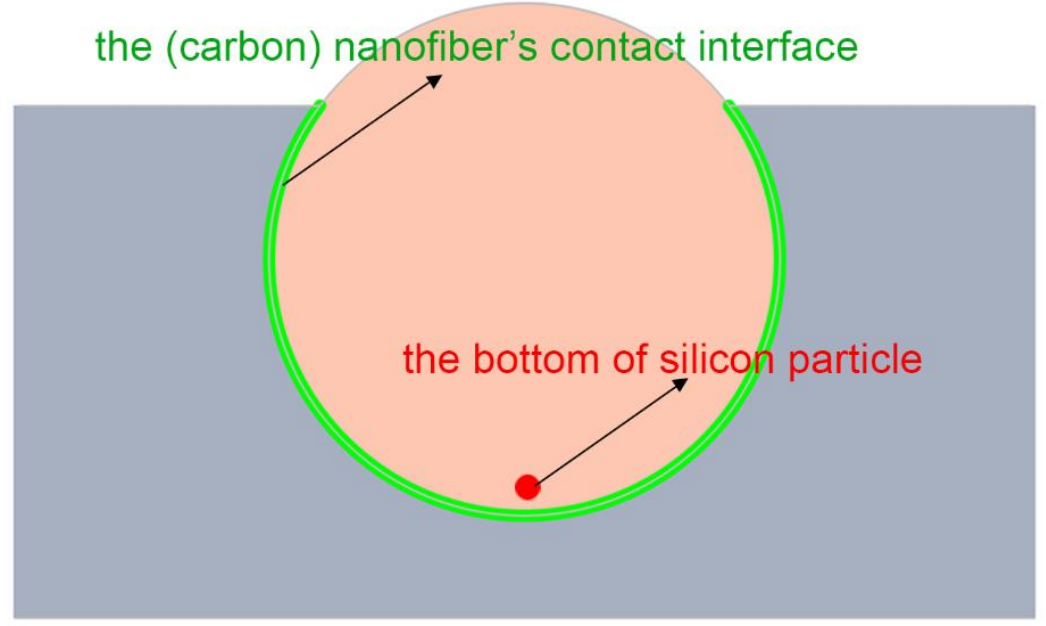

Figure S6. Schematic diagram of the two positions of 'the nanofiber's contact interface' and 'the bottom of the silicon nanoparticle'. 

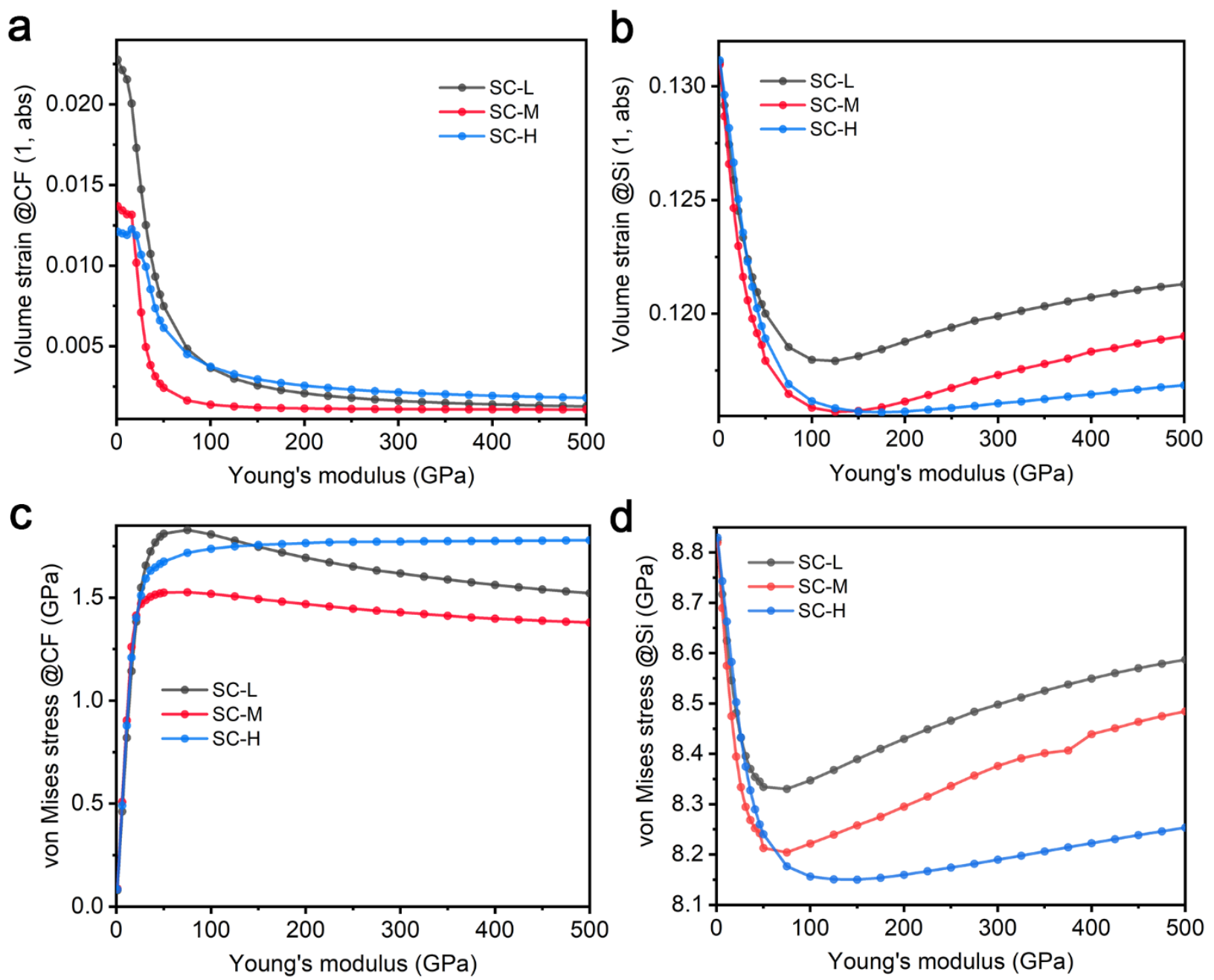

Figure S7. The quantitative analysis of Young's Modulus under over-discharging conditions. (a) The average volume strain at the nanofiber's contact interface, and (b) the volume strain at the bottom of the silicon nanoparticle. (c) The average von Mises stress at the nanofiber's contact interface, and (d) the von Mises stress at the bottom of the silicon nanoparticle. 

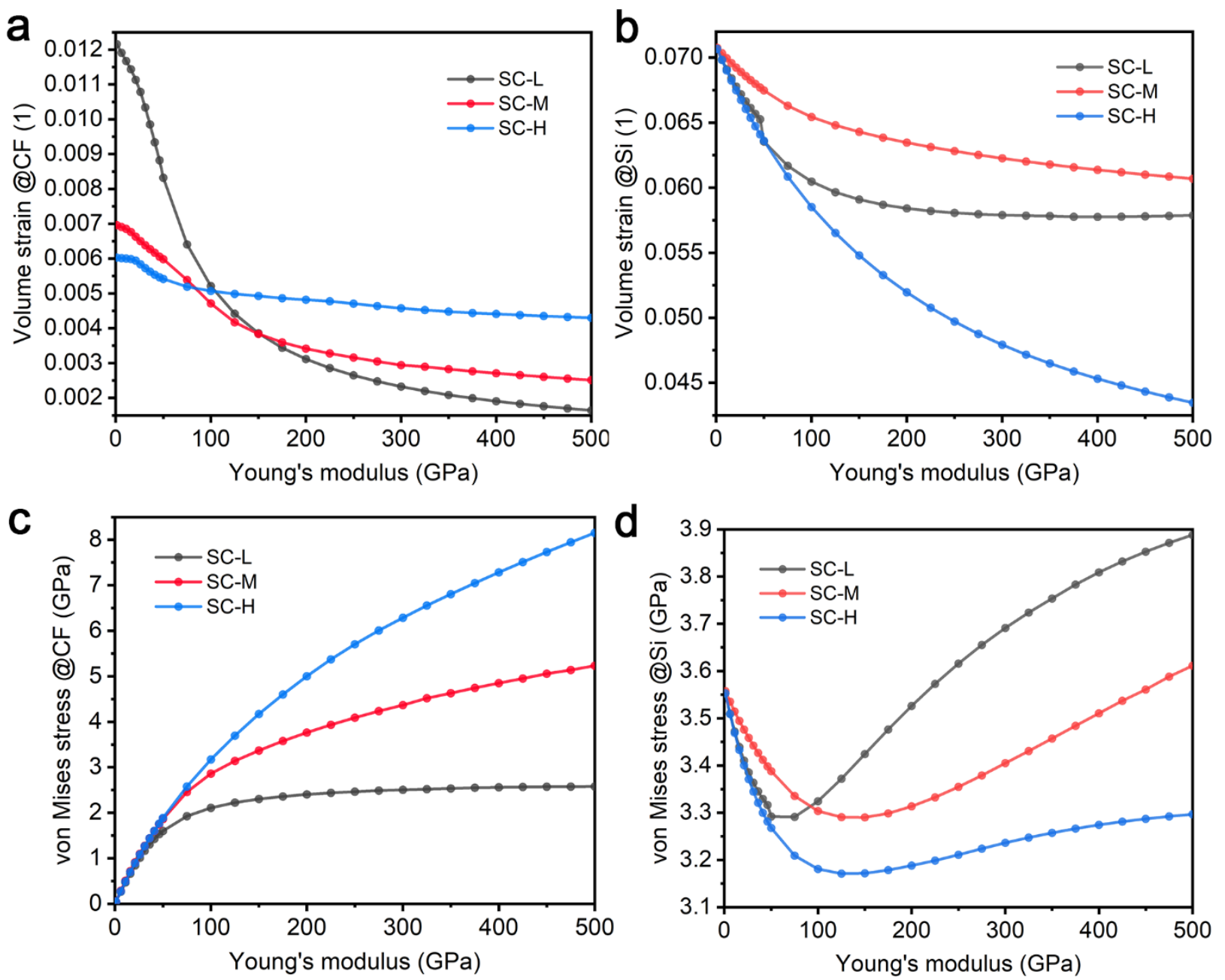

Figure S8. The quantitative analysis of Young's Modulus under charging conditions. (a) The average volume strain at the nanofiber's contact interface, and (b) the volume strain at the bottom of the silicon nanoparticle. (c) The average von Mises stress at the nanofiber's contact interface, and (d) the von Mises stress at the bottom of the silicon nanoparticle. 

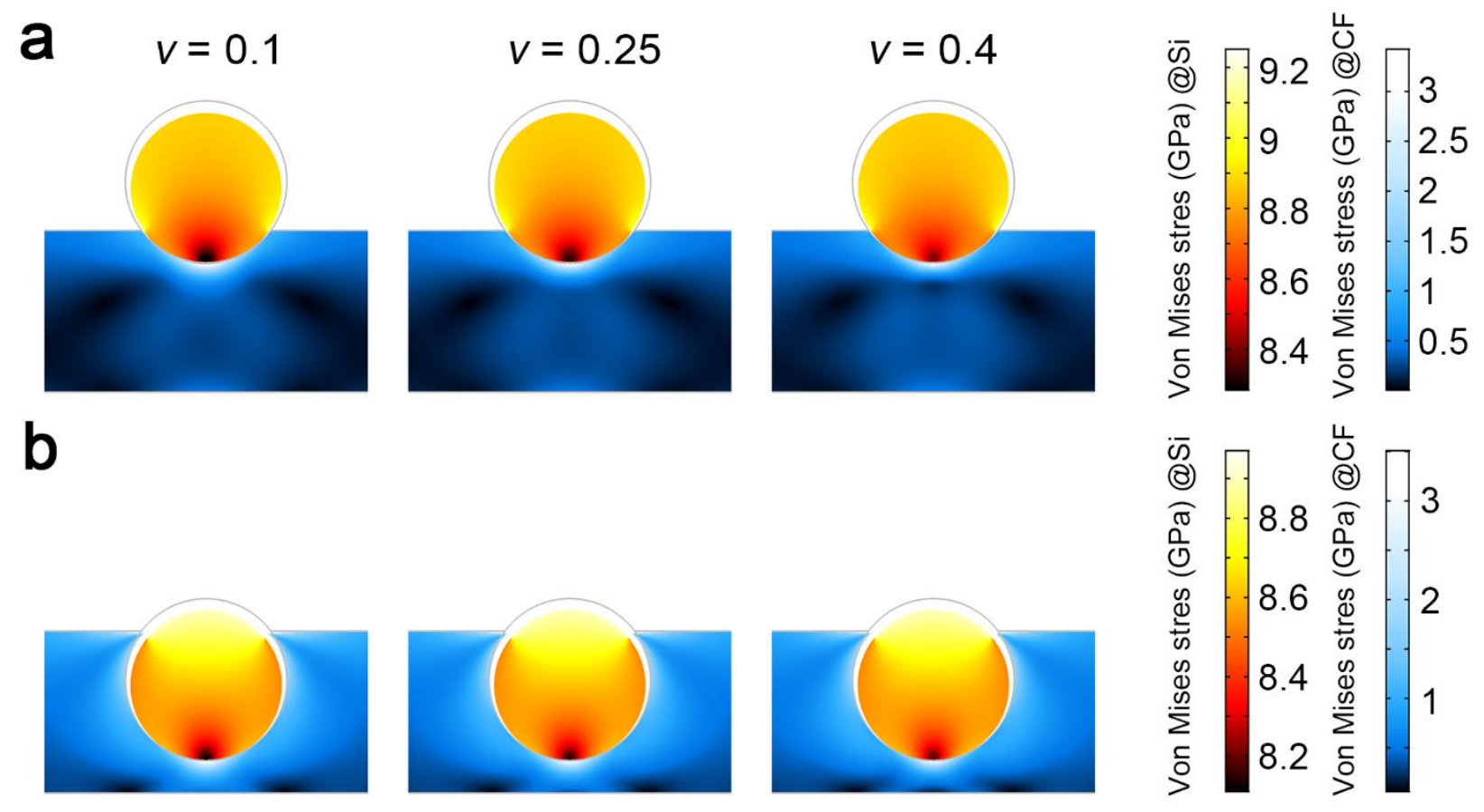

Figure S9. Mechanical properties study of Poisson's ratio $(v)$ of carbon nanofiber under overdischarging conditions. The distribution of von Mises stress in (a) SC-L and (b) SC-H, under Poisson's ratios ranging from 0.1 to 0.4 . 

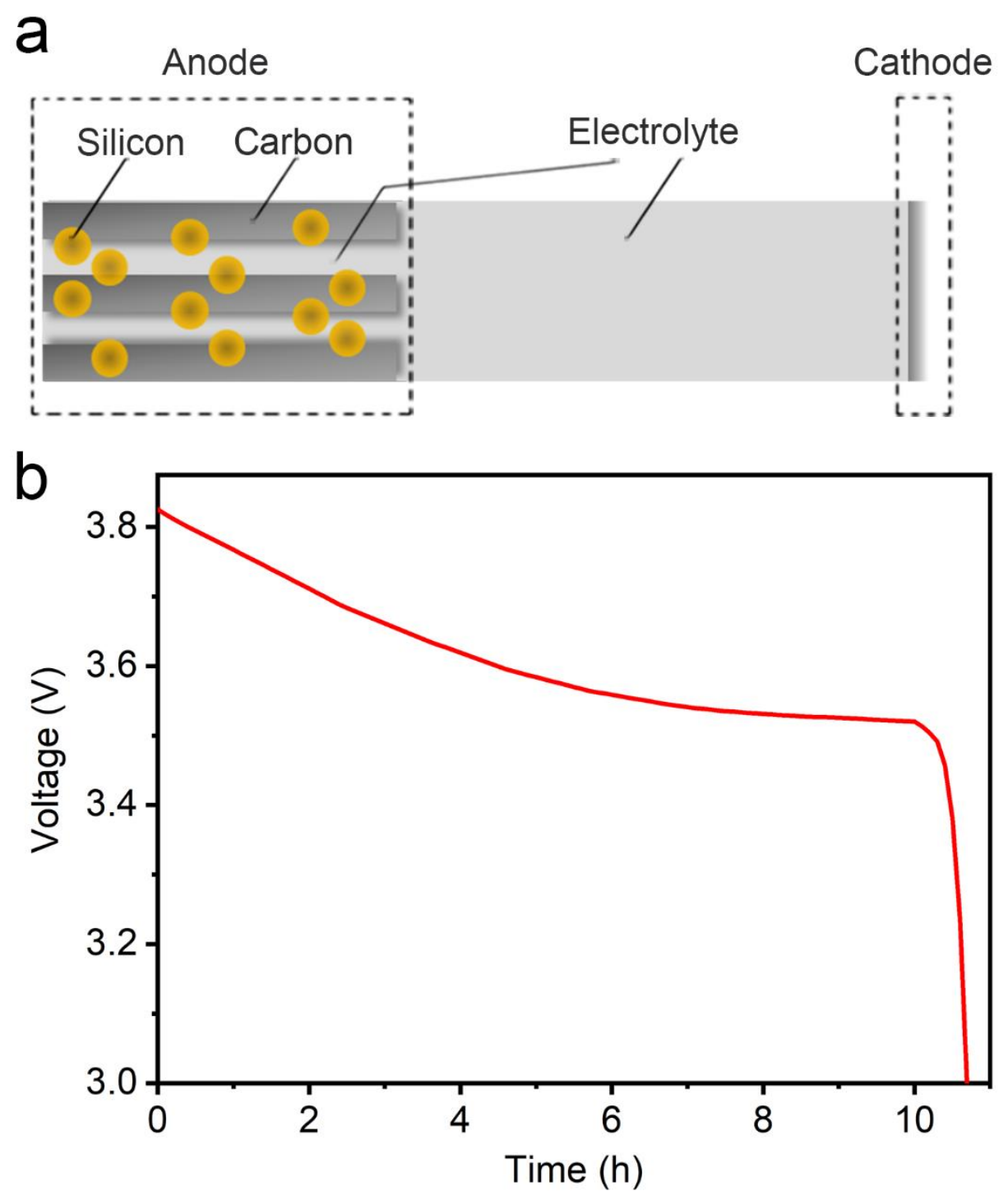

Figure S10. Electrochemical model and simulated discharging curve. (a) Two-dimensional battery model. (b) The simulated discharging curve of the battery model in the electrochemical simulation. 

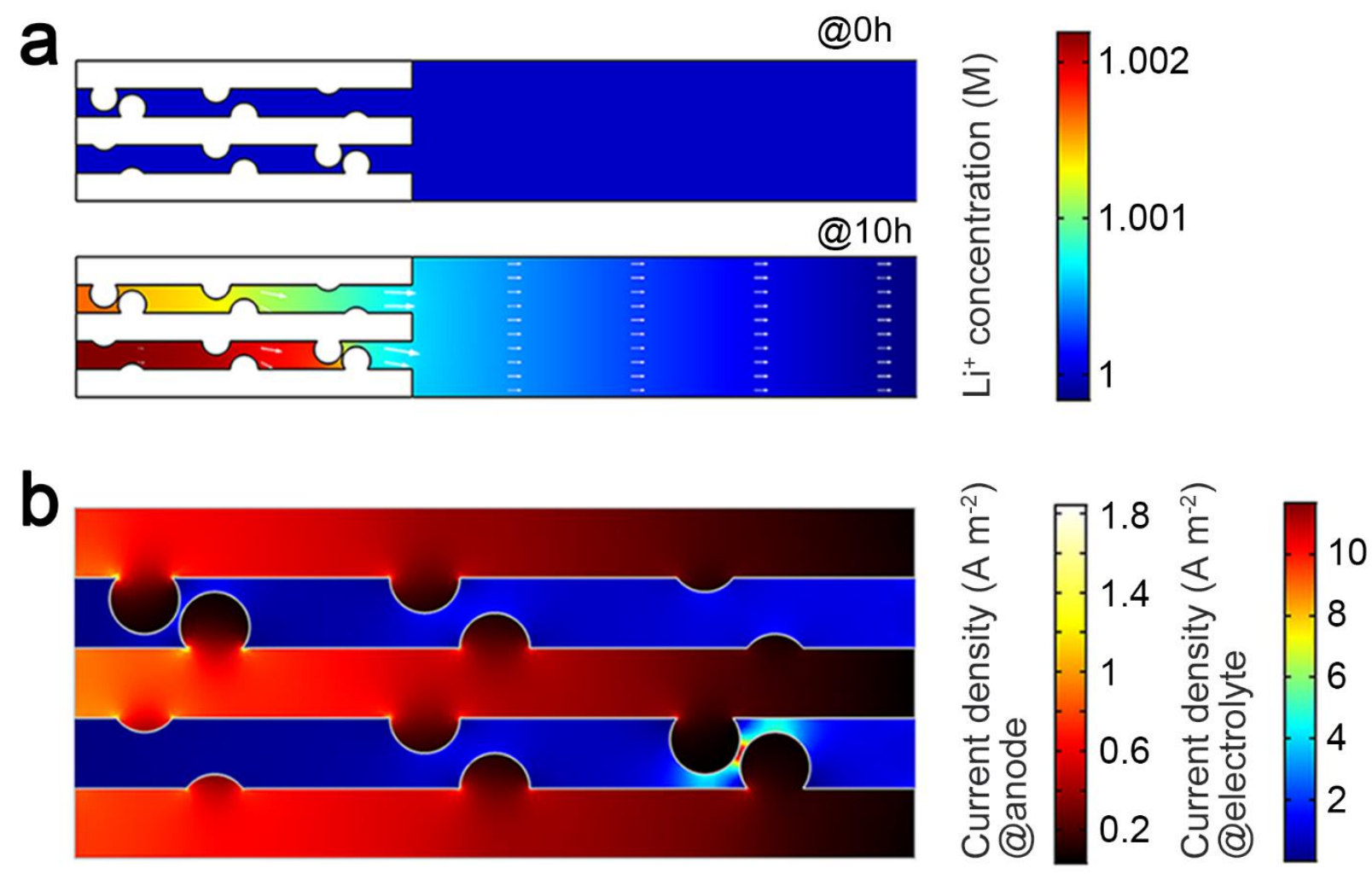

Figure S11. Electrochemical simulation results. (a) The distribution of electrolyte salt concentration at the discharging time of $0 \mathrm{~h}$ and $10 \mathrm{~h}$. (b) The distribution of current density in electrode and electrolyte at the discharging time of $10 \mathrm{~h}$. 
Table S1. Formula form of strain and stress of electrochemical and thermal models ${ }^{1-4}$

\begin{tabular}{ccc}
\hline & Strain & Stress \\
\hline Electrochemical model & $\varepsilon_{e}=\beta\left(I-I_{r e f}\right)=\beta \Delta I^{1}$ & $\sigma_{e}=E_{e} \cdot \beta \Delta I^{2}$ \\
Thermal model & $\varepsilon_{t}=\alpha\left(T-T_{r e f}\right)=\alpha \Delta T^{3}$ & $\sigma_{t}=E_{t} \cdot \alpha \Delta T^{4}$ \\
\hline
\end{tabular}


Table S2. Key parameters in the mechanical and electrochemical simulations

\begin{tabular}{|c|c|c|c|c|}
\hline Model & Parameter & Value & Unit & Definition \\
\hline \multirow{5}{*}{ 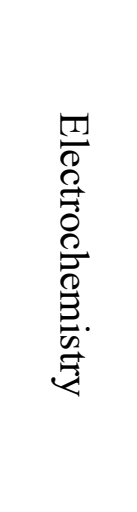 } & $i_{0, \text { ref_anode }}$ & 0.96 & $\mathrm{~A} \mathrm{~m}^{-2}$ & Reference exchange current density of anode \\
\hline & $i_{0, \text { ref_cathode }}$ & 1.72 & $\mathrm{~A} \mathrm{~m}^{-2}$ & Reference exchange current density of cathode \\
\hline & $\alpha_{a}$ & 0.5 & 1 & Anodic transfer coefficient \\
\hline & $\alpha_{c}$ & 0.5 & 1 & Cathodic transfer coefficient \\
\hline & $c_{l, r e f}$ & 1000 & $\mathrm{~mol} \mathrm{~m}^{-3}$ & Electrolyte reference concentration \\
\hline \multirow{13}{*}{ 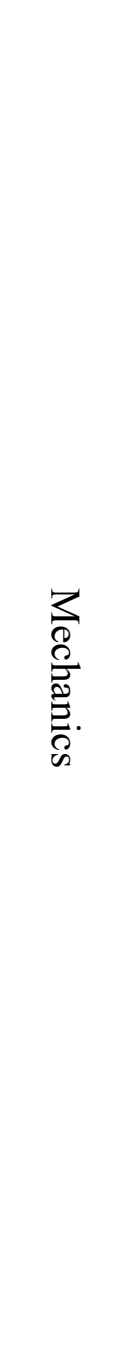 } & $T_{r e f}$ & 293.15 & $\mathrm{~K}$ & Reference temperature \\
\hline & $\alpha_{\text {carbon }}$ & $1 \cdot 10^{-6}$ & $\mathrm{~K}^{-1}$ & Thermal expansion coefficient of carbon \\
\hline & $\rho_{\text {carbon }}$ & 1.7 & $\mathrm{~g} \mathrm{~cm}^{-3}$ & Density of carbon \\
\hline & $E_{\text {carbon }}$ & 230 & $\mathrm{GPa}$ & Young's modulus of carbon \\
\hline & $v_{\text {carbon }}$ & 0.3 & 1 & Poisson's ratio of carbon \\
\hline & $\alpha_{\text {silicon }}$ & $9 \cdot 10^{-5}$ & $\mathrm{~K}^{-1}$ & Thermal expansion coefficient of silicon \\
\hline & $\rho_{\text {silicon }}$ & 2.33 & $\mathrm{~g} \mathrm{~cm}^{-3}$ & Density of silicon \\
\hline & $E_{\text {silicon }}$ & 140 & $\mathrm{GPa}$ & Young's modulus of silicon \\
\hline & $v_{\text {silicon }}$ & 0.265 & 1 & Poisson's ratio of silicon \\
\hline & $\sigma_{t}$ & 800 & $\mathrm{MPa}$ & Tensile strength \\
\hline & $\sigma_{s}$ & 1000 & $\mathrm{MPa}$ & Shear strength \\
\hline & $G_{c t}$ & 9.69 & $\mathrm{~kJ} \mathrm{~m}^{-2}$ & Tensile energy release rate \\
\hline & $G_{c s}$ & 17.19 & $\mathrm{~kJ} \mathrm{~m}^{-2}$ & Shear energy release rate \\
\hline
\end{tabular}




\section{References}

(1) Yang, H.; Fan, F.; Liang, W.; Guo, X.; Zhu, T.; Zhang, S. A Chemo-Mechanical Model of Lithiation in Silicon. J. Mech. Phys. Solids 2014, 70, 349-361.

(2) Xie, H.; Zhang, Q.; Song, H.; Shi, B.; Kang, Y. Modeling and in situ Characterization of Lithiation-induced Stress in Electrodes During the Coupled Mechano-Electro-Chemical Process. J. Power Sources 2017, 342, 896-903.

(3) Wang, M.; Xiao, X. Investigation of the Chemo-Mechanical Coupling in Lithiation/delithiation of Amorphous Si through Simulations of Si Thin Films and Si Nanospheres. J. Power Sources 2016, 326, 365-376.

(4) Cui, D.; Cheng, M. Thermal Stress Modeling of Anode Supported Micro-Tubular Solid Oxide Fuel Cell. J. Power Sources 2009, 192, 400-407. 